\title{
A POSSIBLE MEANS OF DIFFERENTIATION BETWEEN CARDIAC DILATATION AND PERICARDITIS WITH EFFUSION
}

\author{
W. J. CALVERT, M.D. \\ COLUMBia, Mo.
}

A study of the mechanies of large dilatations of the heart shows that in such conditions the right lobe of the liver is actually displaced downward, if displaced at all; but owing to displacement of the bony thorax an apparently normal or elevated position of the liver may be found. This apparently high position of the liver is likely to be found in mitral stenosis with high degree of dilatation. Here the lower anterior and lateral border of the lung is pulled upward, giving a very narrow band of relative lung-liver dulness. In large hearts the upper border of this dulness may be at the fourth rib.

In pericarditis with effusion the mechanical effect is to depress the right lobe of the liver without producing the apparent elevation; also to give a narrow band of relative lung-liver dulness, which is in a lower position than that found in dilatation of the heart. In pericarditis the beginning of the relative dulness over the right lobe of the liver may be below the sixth rib. In cardiac dilatation an apparent elevation, in pericarditis an apparent depression, of the right lobe of the liver may be found. A detailed account of the mechanics of these movements will be publisher later. As the mechanics of the two lesions are so different the recognition of the effects produced on the position of the liver should be of clinical value, save in very fat individuals, on whom it may be almost impossible to map out liver dulness.

In order that this point in differential diagnosis may be of greatest clinical value, an accurate knowledge of the normal position of the liver in each type of thorax is to be desired.

The point is: In cardiac dilatation high liver dulness is present; in pericarditis with effusion low liver dulness; and in each a narrow band of relative lung-liver dulness. 